\title{
Capacity Markets versus Energy-Only Markets: a Comparison under Increasing Wind Penetration
}

\begin{abstract}
We compare the ability to support resource adequacy and revenue sufficiency under two market designs (a capacity market and an energy-only market) and increasing levels of wind energy penetration. An ideal seven node network with six generators and ten transmission lines provides a test system for our analysis. Simulations of power market outcomes are evaluated in terms of capacity expansion and revenue sufficiency.
\end{abstract}

\section{Introduction}

Maintaining long-run generation adequacy and system reliability represents a critical challenge in electric power systems. However, markets do not necessarily provide for the adequate level of generation capacity. During periods of scarcity in which capacity resources are fully utilized, wholesale prices should rise to a level that allow generators to recover their investment costs. However, the existence of demand-side flaws (i.e., lack of consumer response to wholesale prices) and price caps aimed at preventing the exercise of market power in most jurisdictions deprive producers of these high prices. This revenue loss, or "missing money", is considered one of the primary causes of underinvestment in generation $[1,2]$.

Several mechanisms have been designed and implemented to address the missing money problem. In the United States, four of the six FERC jurisdictional wholesale electricity markets (PJM, ISO New England, MISO and New York ISO) operate centralized forward capacity markets in which the system operator sets a generation capacity target and organizes its procurement. The required amount of capacity is contracted between load serving entities and capacity suppliers years and/or months before delivery. Capacity markets are organized as auctions, and the price of the capacity supplied is determined by the settlement between a capacity demand curve that reflects the required reserve margin and the bids of the capacity suppliers. Forward capacity markets in the U.S. RTOs differ with regard to products, capacity procurement methods, and auction procedures. Further, in ISO New
England capacity suppliers are required to sell financial call options to customers when the energy price rises above a pre-agreed strike price, making this design similar to a reliability options model $[3,4]$. CAISO and SPP impose resource adequacy requirements on load serving entities in their footprint, but do not run a centralized capacity market [5].

In contrast, ERCOT opted for an energy and operating reserves market (often referred to as an "energy-only" market design [6]), where energy prices may increase up to $\$ 9000 / \mathrm{MWh}$ during periods of scarcity. A dynamic operating reserve demand curve (ORDC) is implemented in the real-time market to determine an hourly reserve price that depends on the amount of reserves available. Since ERCOT currently does not co-optimize provision of energy and operating reserves in real time, the operating reserve price is derived ex post, and added to the real-time energy price to provide additional revenues to cover fixed costs. It should be noted that energy and/or ancillary service prices surpass the variable cost of the most expensive resources during periods of scarcity even in RTOs that run capacity markets [5, 7]; however, in these jurisdictions scarcity prices are set administratively set at a lower level than the value of lost load (VOLL).

Ensuring resource adequacy and system reliability is particularly challenging in a setting where electricity prices are at historic lows because of declining natural gas prices and increasing penetration of renewable energy sources with zero or low marginal cost. Wholesale electricity prices have dropped on an average annual basis and inframarginal rents in energy markets (through which baseload resources have traditionally recovered a large fraction of their costs, see Figure 1) have declined. Capacity factors for thermal generators have also decreased, further reducing revenues for those plants. As a result of these trends, there is concern that generating firms may fail to recover their capital and operating costs in the long run, preventing future investment in capacity resources that are needed to maintain system reliability.

Under increasing renewable energy penetration, which market design supports resource adequacy while providing sufficient revenues for resources to recover their capital costs? We analyze the performance of capacity markets and energy-only markets under 


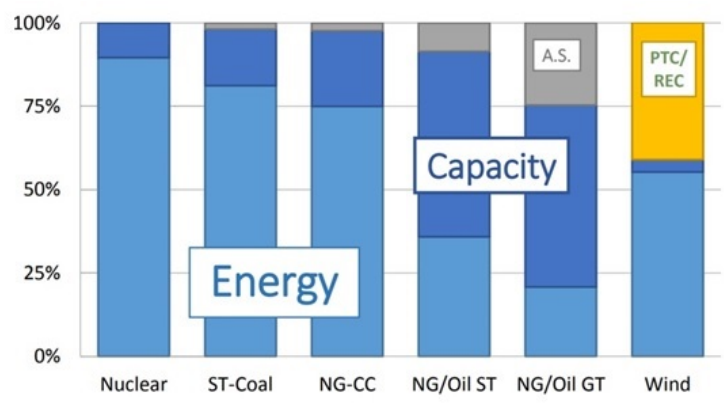

Figure 1. Revenue Source by Technology in NY, NE, and PJM [8]

increasing wind penetration, and uncertainty in wind generation and natural gas prices. First, we formulate a three-stage equilibrium model (for the capacity market design) and a mixed integer program (for the energy-only market design) that determine optimal capacity expansion decisions, and hourly commitment and dispatch decisions. Second, we present impacts of increasing wind penetration under uncertainty on capacity expansion and generator profitability for an ideal seven node network.

\section{Contribution to the literature}

Papers in the literature have compared market designs that are intended to support resource adequacy using various modeling approaches. [9, 10] adopt a system dynamic approach, [11, 12, 13] rely on an optimization approach, while [14, 15] use agent-based simulation models. Our analysis is closely related to studies adopting an optimization approach, and contributes to this strand of literature for several reasons. First, while earlier papers assume a centralized dispatch setting, an equilibrium modeling approach allows for better characterization of individual generation expansion decisions, rather than system plans. For the capacity market design, we present a three-stage equilibrium model that determines optimal capacity expansion decisions, and hourly commitment and dispatch decisions. The three stages represent 1) the capacity market, 2) the day-ahead energy market, and 3) the real-time energy market. To enable computational tractability, we solve the model as a S-adapted open-loop game [16]: all decisions are made simultaneously for all time periods, and market participants adapt their decisions to the sample path of the stochastic variables, without observing what other market participants do when time unfolds. Equilibria for S-adapted open-loop models may be computed through non-linear complementarity problems or variational inequality schemes, which are computationally feasible using current solvers, like PATH. To investigate the impacts of increasing wind penetration in an energy-only market structure, we present a mixed-integer program including a real-time ORDC price adder to determine expansion, commitment and dispatch decisions. We are reformulating this optimization problem as a stochastic equilibrium model, in line with the approach for the capacity market design.

As a second contribution, our analysis accounts for transmission constraints, which are typically disregarded in market design comparisons (e.g., [17]). As unexpected changes in generation become more frequent due to increased variable output, transmission constraints are expected to bind more often, yielding dispatch solutions that may be very different from unconstrained solutions. Thus, our analysis allows for a more accurate representation of the impacts of network constraints, relative to the existing literature.

Finally, earlier studies consider only one source of uncertainty at a time (typically, wind generation). In contrast, we include sources of uncertainty on both the demand and supply side (natural gas prices, demand and wind generation).

\section{Methodology}

Under each market design, we consider four scenarios of wind penetration (no wind, 10\%, 20\% and $30 \%$, where $10 \%$ represents the share of wind power capacity in the test system); for each scenario, we solve the model for four different 24-hour periods of the year representing low and high conditions for natural gas price and wind generation (Figure 2).

\subsection{Capacity market design (CM)}

We present a three-stage equilibrium model that determines optimal capacity expansion decisions, as well as hourly commitment and dispatch decisions. The model is solved using the S-adapted open-loop information structure [16], which assumes that market participants adapt their decisions to the sample path of a stochastic variable, but do not observe other participants actions as time unfolds. Possible realizations of the stochastic variables are described by the event tree in Figure 2, and each path along the tree corresponds to a set of conditions with associated probability.

Real-Time Market. The real-time energy market allows market participants to buy and sell electricity and operating reserves to balance supply and demand during the actual operating day. The auctions act as an adjustment to the day-ahead market. Real-time supply is updated from the scheduled day-ahead commitment to 


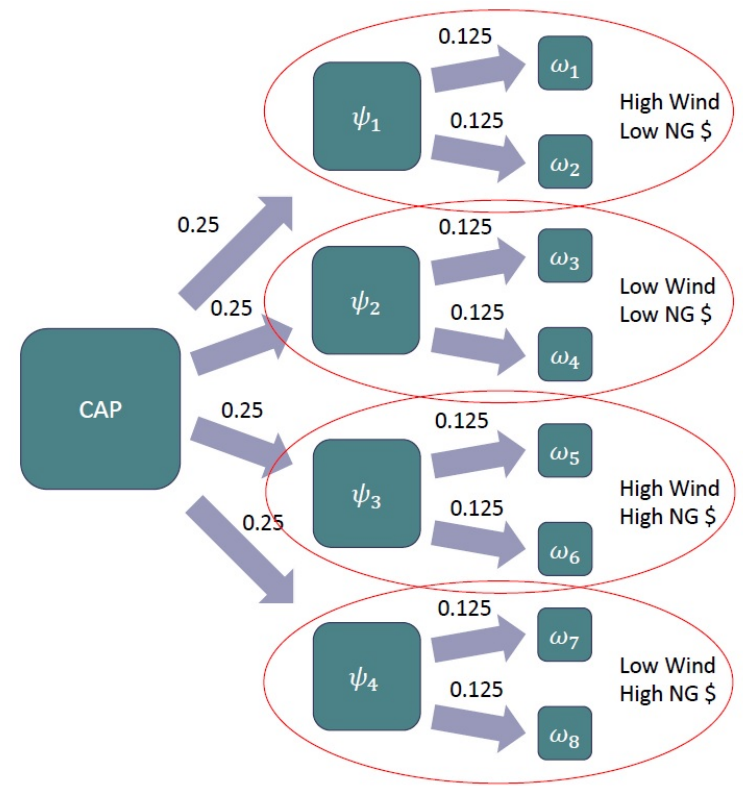

Figure 2. Scenario Tree

account for changes in the actual demand, and determine real-time locational marginal prices (LMPs) for each node in network. We assume that real-time markets are run hourly. ${ }^{1}$ Market players include fast-start generators, slow-start generators, wind generators, and the system operator.

(i) Fast-start generator $j \in J_{i}^{f}$ at node $i$ seeks to maximize profits by choosing the amount of real-time power to generate $\left(x_{i j h}^{\omega}\right)$, and the amount of spinning and non-spinning reserves to provide $\left(s_{i j h}^{\omega}, n s_{i j h}^{\omega}\right)$, where $\omega$ represents the real-time condition, $i$ the node, $j$ the generator, and $h$ the hour. Day-ahead market decisions are indexed by $\psi$, capacity market decisions are given in the real-time stage, and real-time generation is an adjustment to the day-ahead schedule. Each generator receives the real-time energy LMP $\left(p e_{i h}^{\omega}\right)$ for this real-time generation at its node, as well as the spinning and non-spinning reserve price $\left(p s_{h}^{\omega}, p n s_{h}^{\omega}\right)$ for the reserves provided to the system. Fast-start generators are assumed to produce at a constant marginal cost $M C_{i j}$, which is calculated using variable fuel and maintenance costs by technology type. Further, total generation by each plant from the day-ahead and real-time markets must be lower than its production limit $\left(K_{i j}+k_{i j}^{N e w}\right)$ and higher than the minimum output level $\left(\underline{\mathrm{K}}_{i j}\right)$. Units are also subject to ramping limits, which constrain how much their output can increase or decrease over time $\left(R U_{i j}\right.$ and $\left.R D_{i j}\right)$. Lastly, spinning and non-spinning reserve offers made by each generator

\footnotetext{
${ }^{1}$ Real-time markets in the U.S. settle every five minutes.
}

are restricted by the maximum percentage of capacity they are allowed to provide for reserves $\left(S P F_{i j}\right.$ and $N S F_{i j}$ ). Each fast-start generator's real-time problem over 24 hours in a day is:

$$
\begin{gathered}
\max _{\substack{x_{i j h}^{\omega}, s_{i j h}^{\omega} \\
n s_{i j h}^{\omega}}} \sum_{h}\left[\left(p e_{i h}^{\omega}-M C_{i j}\right) \cdot x_{i j h}^{\omega}+\left(p s_{h}^{\omega}+p n s_{h}^{\omega}\right) \cdot s_{i j h}^{\omega}\right. \\
+p n s_{h}^{\omega} \cdot n s_{i j h}^{\omega}
\end{gathered}
$$

subject to:

$$
\begin{gathered}
\left(x_{i j h}^{\psi}+x_{i j h}^{\omega}\right)+s_{i j h}^{\omega}+n s_{i j h}^{\omega} \leq\left(K_{i j}+k_{i j}^{N e w}\right), \quad \forall h \in H \\
x_{i j h}^{\psi}+x_{i j h}^{\omega} \geq \underline{\mathrm{K}}_{i j}, \quad \forall h \in H \\
\left(x_{i j h}^{\psi}+x_{i j h}^{\omega}\right)-\left(x_{i j h-1}^{\psi}+x_{i j h-1}^{\omega}\right)+s_{i j h}^{\omega}+n s_{i j h}^{\omega} \leq R U_{i j}, \\
\forall h \in H
\end{gathered}
$$

$\left(x_{i j h}^{\psi}+x_{i j h}^{\omega}\right)-\left(x_{i j h-1}^{\psi}+x_{i j h-1}^{\omega}\right) \geq-R D_{i j}, \quad \forall h \in H$

$s_{i j h}^{\omega} \leq\left(K_{i j}+k_{i j}^{N e w}\right) \cdot S P F_{i j}, \quad \forall h \in H$

$n s_{i j h}^{\omega} \leq\left(K_{i j}+k_{i j}^{N e w}\right) \cdot N S F_{i j}, \quad \forall h \in H$

$s_{i j h}^{\omega} \geq 0, \quad n s_{i j h}^{\omega} \geq 0, \quad \forall h \in H$

$x_{i j h}^{\psi}+x_{i j h}^{\omega} \geq 0, \quad \forall h \in H$

(ii) Unlike fast-start generators, slow-start generators cannot offer non-spinning reserves into the market. Hence, the problem solved by $j \in J_{i}^{S}$ is similar to the one presented above, but does not include non-spinning reserve variables.

(iii) Wind generator $j \in J_{i}^{w}$ solves the following problem:

$$
\max _{x_{i j h}^{\omega}} \sum_{h}\left[\left(p e_{i h}^{\omega}-M C_{i j}\right) \cdot x_{i j h}^{\omega}+P T C \cdot x_{i j h}^{\omega}\right]
$$

subject to $\left(x_{i j h}^{\psi}+x_{i j h}^{\omega}\right) \leq W_{i j h}^{\omega}, \quad \forall h \in H$

$$
x_{i j h}^{\psi}+x_{i j h}^{\omega} \geq 0, \quad \forall h \in H
$$

where generation must be lower than the real-time forecast production, which differs by hour and condition (i.e. $\left.W_{i j h}^{\omega}\right)$. Wind producers also receive a production tax credit for the energy they produce (PTC).

(iv) The system operator's problem is:

$$
\max _{y_{i h}^{\omega}} \sum_{h} \sum_{i} p e_{i h}^{\omega} \cdot y_{i h}^{\omega}
$$




$$
\begin{gathered}
\text { subject to } \sum_{i}\left(y_{i h}^{\psi}+y_{i h}^{\omega}\right)=0, \quad \forall h \in H \\
-\sum_{i} P T D F_{i l}\left(y_{i h}^{\psi}+y_{i h}^{\omega}\right) \leq T_{l}, \quad \forall l \in L, \forall h \in H \\
-\sum_{i} P T D F_{i l}\left(y_{i h}^{\psi}+y_{i h}^{\omega}\right) \geq-T_{l}, \quad \forall l \in L, \forall h \in H
\end{gathered}
$$

where $y_{i h}^{\omega}$ represents the net injection adjustment into node $i$ during hour $h$ and condition $\omega$.

(v) The consumer problem in real time is:

$$
\max _{e s_{i h}^{\omega} S r s_{h}^{\omega}, n s r s_{h}^{\omega}} \sum_{h}\left[\sum_{i}\left(E O C-p e_{i h}^{\omega}\right) \cdot\left(D_{i h}^{\omega}-e s_{i h}^{\omega}\right)+\left(S C-p s_{h}^{\omega}\right) .\right.
$$$$
\left.\cdot\left(S_{h}^{\omega}-s r s_{h}^{\omega}\right)+\left(S C-p n s_{h}^{\omega}\right) \cdot\left(S_{h}^{\omega}+N S_{h}^{\omega}-n s r s_{h}^{\omega}\right)\right]
$$

subject to $0 \leq e s_{i h}^{\omega} \leq D_{i h}^{\omega}, \quad \forall i \in I, \forall h \in H$

$$
s r s_{h}^{\omega} \geq 0, n s r s_{h}^{\omega} \geq 0, \quad \forall h \in H
$$

where $e s_{i h}^{\omega}$ is the energy scarcity (lost load) at $i$ during hour $h$, which we assume priced at an energy offer cap (EOC) lower than VOLL, and $s r s_{h}^{\omega}$ and $n s r s_{h}^{\omega}$ are the spinning and non-spinning reserve scarcity in hour $h$, which we assume are priced at the administratively set scarcity penalty.

(vi) Finally, the hourly real-time market clearing conditions for energy, spinning reserves, and non-spinning reserves are, respectively:

$$
\begin{gathered}
\sum_{j \in J_{i}}\left(x_{i j h}^{\psi}+x_{i j h}^{\omega}\right)+y_{i h}^{\psi}+y_{i h}^{\omega}=D_{i h}^{\omega}-e s_{i h}^{\omega} \quad\left(p e_{i h}^{\omega}\right) \\
\forall i \in I, \forall h \in H \\
\sum_{i} \sum_{J_{i}^{h t}} s_{i j h}^{\omega}=S_{h}^{\omega}-s r s_{h}^{\omega} \quad\left(p s_{h}^{\omega}\right), \quad \forall h \in H \\
\sum_{i} \sum_{J_{i}^{f}}\left(s_{i j h}^{\omega}+n s_{i j h}^{\omega}\right)=S_{h}^{\omega}+N S_{h}^{\omega}-n s r s_{h}^{\omega} \quad\left(p n s_{h}^{\omega}\right), \\
\forall h \in H
\end{gathered}
$$

Day-Ahead Market. The day-ahead energy market allows market participants to commit to buying or selling electricity one day before actual power is dispatched. This creates a schedule of electricity production to be delivered for each hour of the following day based on load and wind forecasts. A day-ahead LMP is established at each node in the network for each hour of the delivery day.

(i) Fast-start generator $j \in J_{i}^{f}$ at node $i$ seeks to maximize total profits by choosing its day-ahead schedule $\left(x_{i j h}^{\psi}\right)$, where $\psi$ represents the day-ahead condition. Capacity market decisions are given in the day-ahead stage. Each generator receives the nodal LMP $\left(p e_{i h}^{\psi}\right)$ for generation at its own node, and the day-ahead schedule is bounded by total capacity. Finally, expected real-time market profits are included in the objective function. The formulation for each generator's day-ahead problem (for each day-ahead scenario $\psi$ ) is presented below:

$$
\begin{aligned}
\max _{x_{i j h}^{\psi}} & \sum_{h}\left[\left(p e_{i h}^{\psi}-M C_{i j}\right) \cdot x_{i j h}^{\psi}\right] \\
& +\mathbb{E}\left\{\operatorname { m a x } _ { \substack { x _ { i j h } ^ { \omega } , s _ { i j h } ^ { \omega } \\
n s _ { i j h } ^ { \omega } } } \sum _ { h } \left[\left(p e_{i h}^{\omega}-M C_{i j}\right) \cdot x_{i j h}^{\omega}\right.\right. \\
& \left.\left.+\left(p s_{h}^{\omega}+p n s_{h}^{\omega}\right) \cdot s_{i j h}^{\omega}+p n s_{h}^{\omega} \cdot n s_{i j h}^{\omega}\right]\right\}
\end{aligned}
$$

(ii) Slow-start generators $j \in J_{i}^{s}$ do not include the real-time non-spinning reserve variable in the real-time expectation.

(iii) Wind generator $j \in J_{i}^{w}$ solves the following problem:

$$
\begin{array}{r}
\max _{x_{i j h}^{\psi}} \sum_{h}\left[\left(p e_{i h}^{\psi}-M C_{i j}\right) \cdot x_{i j h}^{\psi}+P T C \cdot x_{i j h}^{\psi}\right] \\
+\mathbb{E}\left\{\max _{x_{i j h}^{\omega}} \sum_{h}\left[\left(p e_{i h}^{\omega}-M C_{i j}\right) \cdot x_{i j h}^{\omega}+P T C \cdot x_{i j h}^{\omega}\right]\right\}
\end{array}
$$

subject to $0 \leq x_{i j h}^{\psi} \leq W_{i j h}^{\psi}, \quad \forall h \in H$

where generation must be lower than an exogenous day-ahead production forecast that differs by hour and condition, $W_{i j h}^{\psi}$.

(v) Finally, the hourly day-ahead market clearing condition for energy is:

$\sum_{j \in J_{i}} x_{i j h}^{\psi}+y_{i h}^{\psi}=D_{i h}^{\psi}\left(p e_{i h}^{\psi}\right), \quad \forall i \in I, \forall h \in H$

We assume that reserves are only provided in real time, hence there is no day-ahead market clearing condition for reserves.

Capacity Market. The capacity market stage allows generators to procure new capacity resources to meet a reserve margin target determined by the system operator. 
We assume that the auctions are run far enough in advance (i.e. 3 year like in the markets run by PJM or NYISO) for generators to physically build new capacity by the delivery year. A vertical demand curve specification, as employed by NE-ISO and MISO, sets the planning reserve margin, which is modeled as a single "target" value used to clear the market.

(i) Fast-start generator $j \in J_{i}^{f}$ at node $i$ offers its existing capacity $\left(K_{i j}\right)$ and any potential new capacity investments $\left(k_{i j}^{N e w}\right)$ into the auction to maximize annualized profits. These include the annual revenue received from the capacity market payments minus the annualized capital cost of building new capacity $\left(K C_{i j}\right)$. Also included are the expected profits received from the day-ahead and real-time markets. In this formulation, $p c$ refers to the market clearing price for capacity, $f_{i j}$ is the capacity owned by $j$ that clears the auction, and $A C_{i j}$ represents the annual depreciation cost of the existing capacity. Additionally, the capacity that clears the auction for all generators is bounded by their total capacity amount. The problem formulation for $j$ at $i$ is:

$$
\begin{array}{rl}
\max _{f_{i j}, k_{i j}^{N e w}} & 365 \cdot p c \cdot f_{i j}-K C_{i j} \cdot k_{i j}^{N e w}-A C_{i j} \\
& +\mathbb{E}\left\{\max _{x_{i j h}^{\psi}} \sum_{h}\left[\left(p e_{i h}^{\psi}-M C_{i j}\right) \cdot x_{i j h}^{\psi}\right]\right. \\
& +\mathbb{E}\left\{\operatorname { m a x } _ { \substack { x _ { i j h } ^ { \omega } , s _ { i j h } ^ { \omega } \\
n s _ { i j h } } } \sum _ { h } \left[\left(p e_{i h}^{\omega}-M C_{i j}\right) \cdot x_{i j h}^{\omega}\right.\right. \\
\left.\left.\left.+\left(p s_{h}^{\omega}+p n s_{h}^{\omega}\right) \cdot s_{i j h}^{\omega}+p n s_{h}^{\omega} \cdot n s_{i j h}^{\omega}\right]\right]\right\}
\end{array}
$$

(ii) Slow-start generators $j \in J_{i}^{s}$ do not include the real-time non-spinning reserve variable in the real-time expectation.

(iii) Wind generator $j \in J_{i}^{w}$ at node $i$ receive a capacity credit $\left(C C_{i j}\right)$ for their offer into the auction:

$$
\begin{array}{r}
\max _{f_{i j}, k_{i j}^{N \text { Now }}} 365 \cdot p c \cdot f_{i j}-A C_{i j}+\mathbb{E}\left\{\operatorname { m a x } _ { x _ { i j h } ^ { \psi } } \sum _ { h } \left[\left(p e_{i h}^{\psi}-M C_{i j}\right)\right.\right. \\
\left.\cdot x_{i j h}^{\psi}+P T C \cdot x_{i j h}^{\psi}\right]+\mathbb{E}\left\{\operatorname { m a x } _ { x _ { i j h } ^ { \omega } } \sum _ { h } \left[\left(p e_{i h}^{\omega}-M C_{i j}\right) \cdot x_{i j h}^{\omega}\right.\right. \\
\left.\left.\left.+P T C \cdot x_{i j h}^{\omega}\right]\right\}\right\} \\
\text { subject to } f_{i j} \leq K_{i j}^{\text {Old }} \cdot C C_{i j}
\end{array}
$$

$f_{i j} \geq 0$,

(iv) The market clearing condition sets an inelastic resource adequacy requirement (TARGET) which consists of a forecasted future peak demand level plus a $10 \%$ reserve margin. The sum of all capacity that clears the market for each generator must equal the resource adequacy target set by the system operator:

$T A R G E T=\sum_{i} \sum_{j} f_{i j} \quad(p c)$,

\subsection{Energy-only market design (ORDC)}

To model the energy-only market structure, we develop and solve a nonlinear optimization model in which the electricity market operator minimizes the total cost of operations over a 24-hour period, including fixed investment costs, operating costs, and the costs associated with lost load (i.e., reserve scarcity) events. Each stage of the optimization is run simultaneously, subject to the physical and inter-temporal constraints for electricity generators (e.g. ramping rates, capacity constraints, etc.), and real-time optimal dispatch solutions represent an adjustment from the day-ahead commitment schedule. In particular, the real-time objective of the system operator's problem is as follows:

$$
\begin{aligned}
& \min \sum_{h}\left[\sum_{i} \sum_{j \in J_{i}} M C_{i j} \cdot x_{i j h}^{\omega}+\sum_{i} E O C \cdot e s_{i h}^{\omega}+R B S_{h}^{\omega}\left[R s_{h}^{\omega}\right]\right. \\
& \left.+R B N S_{h}^{\omega}\left[R n s_{h}^{\omega}\right]-\sum_{i} \sum_{j \in J_{i}^{\omega}} P T C \cdot x_{i j h}^{\omega}\right]
\end{aligned}
$$

EOC represents the costs associated to energy scarcity, or the value of lost load. $R B S$ and $R B N S$ represent instead the societal benefits generated through the provision of spinning and non-spinning reserves by thermal generators. ${ }^{2}$ These benefits are defined by hourly operating reserve demand curve (ORDC) functions, which are solved directly within the model. The calculation of ORDCs merits further discussion. An ORDC is constructed for each of four seasons and six time-of-day blocks (each consisting of four hours), for a total of 24 curves. Mean and standard deviation of the hourly system-wide error (i.e., deviation between hour-ahead and actual reserves) in 2011 and 2012 are publicly available from ERCOT, the only U.S. market implementing ORDC curves [19]. Since the error is assumed to be normally distributed [19], using historical

\footnotetext{
${ }^{2} \mathrm{We}$ assume that wind generators cannot provide operating reserves for reliability purposes [18].
} 
data we calculate the loss of load probability for the non-spinning and spinning reserves as follows:

$$
\begin{aligned}
& \operatorname{LOLP}\left(\mu_{h, s}, \sigma_{h, s}, s+n s-X\right) \\
& \quad=1-\operatorname{CDF}\left(\mu_{h, s}, \sigma_{h, s}, s+n s-X\right) \\
& \operatorname{LOLP}\left(\mu_{h, s}, \sigma_{h, s}, s-X\right) \\
& =1-\operatorname{CDF}\left(\frac{\mu_{h, s}}{2}, \frac{\sigma_{h, s}}{\sqrt{2}}, s-X\right)
\end{aligned}
$$

where $h$ indicates an hour in one of the six time-of-day blocks, $s$ denotes season, CDF is the cumulative density function of the normal distribution with mean $\mu$ and standard deviation $\sigma$, and $\mathrm{X}$ represents the minimum contingency level for the system (assumed to be 200 MW, which is $10 \%$ of the peak load). The LOLP represents the probability that an unforeseen shortfall in capacity (or demand increase) exceeds the level of reserves. We adjust mean and standard deviation using a scaling factor for wind penetration, as in [12]. Assuming that lambda is the marginal cost of generation from a NGCC unit (\$46.50/MWh) This is used to obtain an adder to the system real-time energy price that quantifies the value of scarce reserve capacity, and is derived from the first order conditions of the two-stage stochastic problem first presented in [6]: ${ }^{3}$

$$
\begin{aligned}
& P_{n s}=0.5 \cdot(\operatorname{VOLL}-\lambda) \\
& \cdot\left(1-C D F\left(\mu_{h, s}, \sigma_{h, s}, s+n s-X\right)\right) \\
& P_{s}=P_{n s}+ 0.5 \cdot \max (\operatorname{VOLL}-\lambda, 0) \\
& \cdot\left(1-C D F\left(\frac{\mu_{h, s}}{2}, \frac{\sigma_{h, s}}{\sqrt{2}}, s-X\right)\right)
\end{aligned}
$$

where VOLL represents the value attributed to consumers to unsupplied energy and is assumed to equal \$9,000/MWh [12]. Note that a different reserve price corresponds to each level of reserves above the minimum contingency level, $s+n s-X$ or $s-X$. The ORDC maps each level of reserves provided in the system to a reserves price. In each hour, the market clearing price for reserves (and adder to the real-time energy price) is determined based on the value of the ORDC curve that corresponds to the level of reserves provided in the system.

\section{Data}

We solve the models on a modified version of the 7-node network presented in [20]. In this test system, six generators are located at separate nodes on the network, which are all connected by transmission constrained

\footnotetext{
${ }^{3}[17]$ provides an analytical derivation of the first order conditions for this problem.
}

lines. There are separate consumer loads at six nodes in the network, leaving one without any demand. This is considered to be the slack bus, or hub node, and is where all power is assumed to flow through. A system operator is modeled to efficiently manage the power flows on the transmission network by accounting for the PTDFs (derived from PLEXOS [20]). A representation of this 7 -node network is presented in Figure 3.

The six generators included in the model represent a nuclear plant, a coal plant, a natural gas combined cycle plant (NGCC), two natural gas combustion turbine (NGCT) plants (which have the same parameters but are located at different nodes) and one wind turbine. Each of these has a different generating capacity (MW), capital cost of construction (in $\$ / \mathrm{kW}$ ), marginal cost of production (in $\$ / \mathrm{MWh}$ ), and lifetime (in years) [12] [21]. The marginal cost of production for each generation technology type was calculated using their average fuel cost (in \$/ton or \$/Mcf) from 2017, heat content (in Btu/lb or Btu/Mcf), heat rate (in Btu/kWh), and variable operations/maintenance costs (in $\$ / \mathrm{MWh}$ ). The coal and nuclear plants are assumed to have been built in the 1970s/1980s, therefore capital cost data comes from older sources to reflect the costs when they were built [22] [23]. Similarly, the NGCC plant was assumed to have been built in the year 2000, and capital costs are from [24].

The $10 \%$ and $20 \%$ levels of the wind penetration scenarios are chosen based on 2017 operating capacity data found from [21]. CAISO has a wind penetration share of about $11 \%$ by capacity, while ERCOT and SPP have the highest levels of wind penetration by capacity (about 19\% and 20\%, respectively). Our highest wind scenario case is assumed to be $10 \%$ higher than the penetration levels of ERCOT and SPP.

\section{Preliminary Results}

Four different scenarios are run for the capacity market and energy-only market models, representing a no-wind setting, a $10 \%$ wind penetration setting, a $20 \%$ wind penetration setting, and a $30 \%$ wind penetration setting. Within each of these scenarios, different conditions are created to represent uncertainty in natural gas prices, wind generation levels, and changes in demand from the day-ahead market to the real-time market. For example, there are two sets of natural gas prices that are assumed in the model, a high price of $\$ 7 / \mathrm{Mcf}$ and a low price of $\$ 3.5 / \mathrm{Mcf}$. The low price is roughly similar to the current cost of natural gas for electricity producers and the high price is about the same as how natural gas costs were in 2007. Additionally, for the $10 \%, 20 \%$, and $30 \%$ wind scenarios, there 


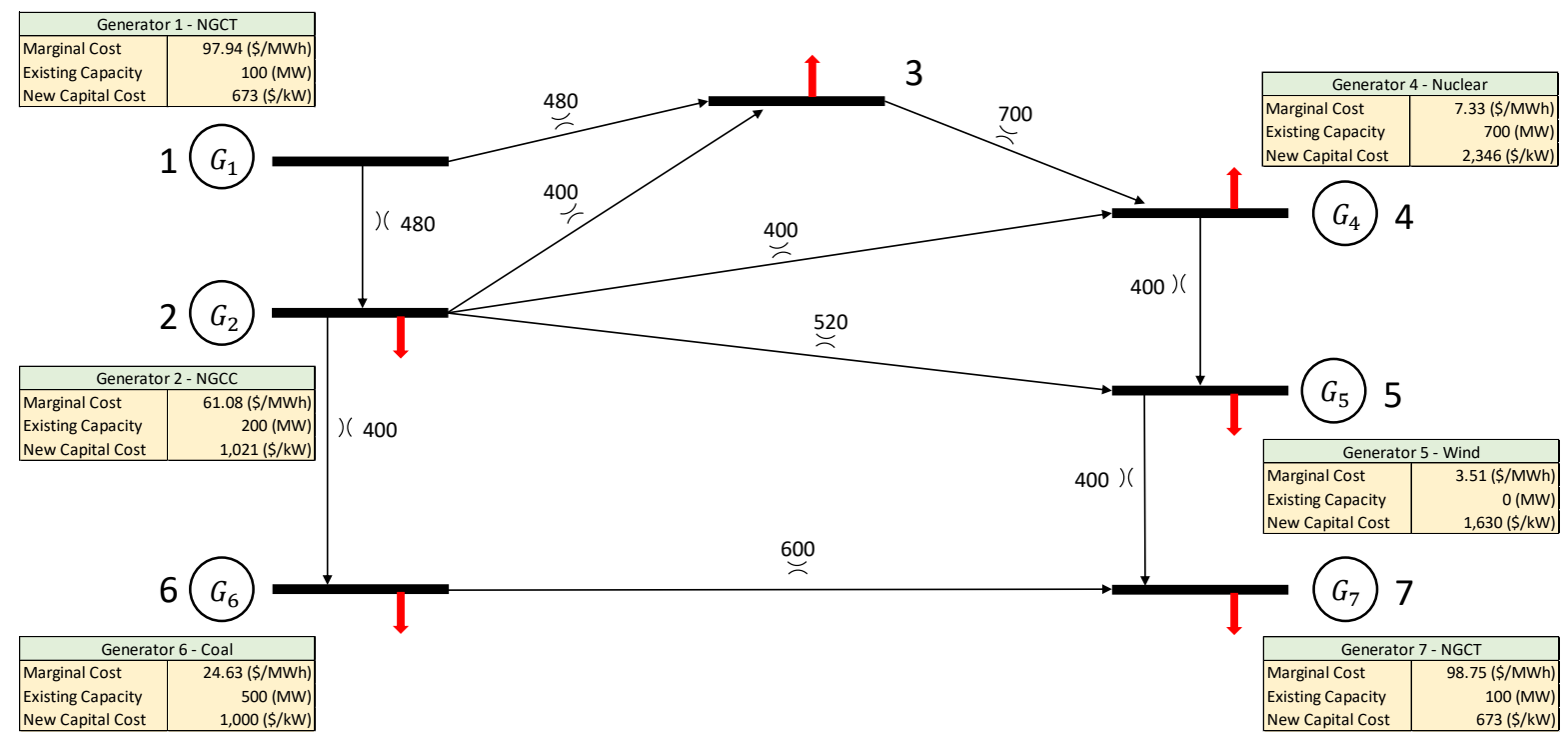

Figure 3. 7-Node Network

is a significant amount of uncertainty related to wind production in the day-ahead market. The real-time market also has uncertainty related to wind production, but at a much lower level than in the day-ahead. Lastly, there is some demand uncertainty included in the model, which accounts for a difference in the day-ahead and real-time loads. Figure 2 shows how we account for uncertainty by having different combinations of natural gas prices and wind generation levels (demand is also uncertain in real-time). Both models are solved in GAMS. The equilibrium problem is solved as a mixed complementarity model (MCP) using the PATH solver, and converges to a local optimum in about a minute. The energy-only model is solved as a nonlinear optimization problem using the CONOPT solver, and converges in about 5 seconds.

Since the decision problems of the market players include expectations of day-ahead and real-time stages, our results should be interpreted as average values. We simulate results for the four wind penetration scenarios, and compare them based on multiple metrics. For example, the amount of new generation capacity added on by thermal generators changes under different levels of wind penetration. Figures $4-6$ show the existing capacity for each generator in the system, as well as the new capacity added on in the capacity market and energy-only market models. As more wind generation enters the network, less capacity needs to be added on by thermal generators. Only the NGCC and NGCT plants are able to add on new capacity in our models. In the energy-only model, the NGCC is the only plant that adds on new capacity. While the capital costs of the NGCC generator is higher than the NGCT generators, the marginal cost of production is lower. Adding on new NGCC capacity would allow the plant to generate more electricity in the day-ahead and real-time markets, reducing the need for more expensive generation from the NGCT plants. Further, the energy-only market model adds on far less total new capacity than the capacity market model. Results presented here assume a moderate level of electricity demand. Given this level of demand, the energy-only market model does not need to add on much new capacity, whereas the capacity market model sets a requirement on how much new capacity needs to be added. In the no-wind case, the capacity model adds $556 \mathrm{MW}$ of total new capacity, while the energy-only model adds $240 \mathrm{MW}$ of total new capacity. In the $10 \%$ wind case, the capacity model adds $499 \mathrm{MW}$ of total new capacity, while the energy-only model adds 180 MW of total new capacity. Lastly, in the $30 \%$ wind case, the capacity model adds 387 MW of total new capacity, while the energy-only model adds $100 \mathrm{MW}$ of total new capacity.

The expected daily real-time generation levels for each plant with non zero generation in the capacity market and energy-only market model are compared across wind cases in Figures 7 and 8. The nuclear plant produces at capacity for all hours of the day 


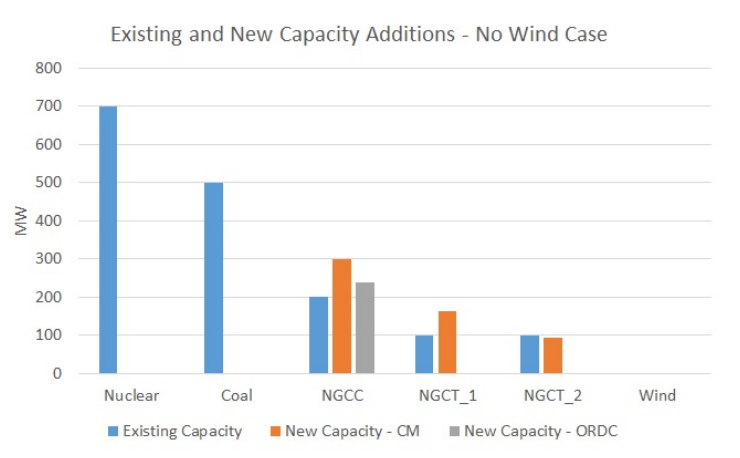

Figure 4. Total Capacity Levels - No Wind Case

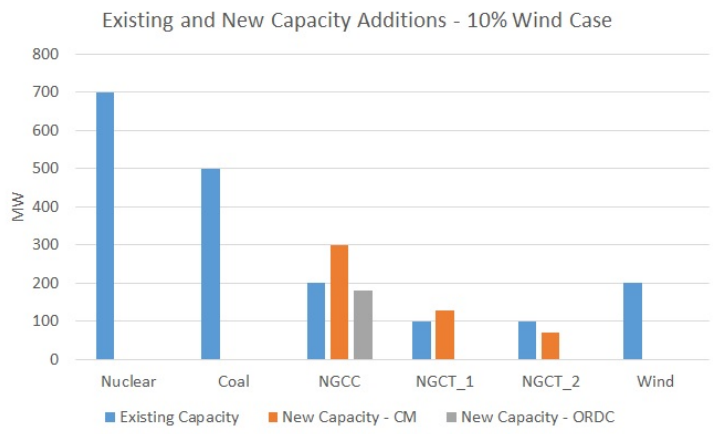

Figure 5. Total Capacity Levels - 10\% Wind Case

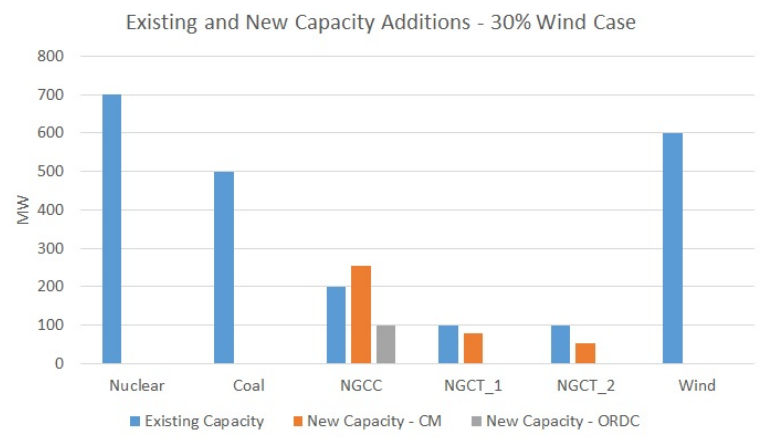

Figure 6. Total Capacity Levels - 30\% Wind Case

in each wind penetration scenario. The coal plant initially produces at capacity, but as wind penetration increases, its production slightly decreases. For the NGCC plant, production decreases steadily as wind generation increases. The reason for this is that wind is displacing coal and NGCC-based generation, as its marginal cost of production is lower. The NGCT plants are used slightly more in the energy-only market model, because the NGCC plant does not add on as much new capacity in the energy-only market model. Therefore, in some hours of the day, the NGCT plants would need to be dispatched in the energy-only model when the NGCC is operating at full capacity.

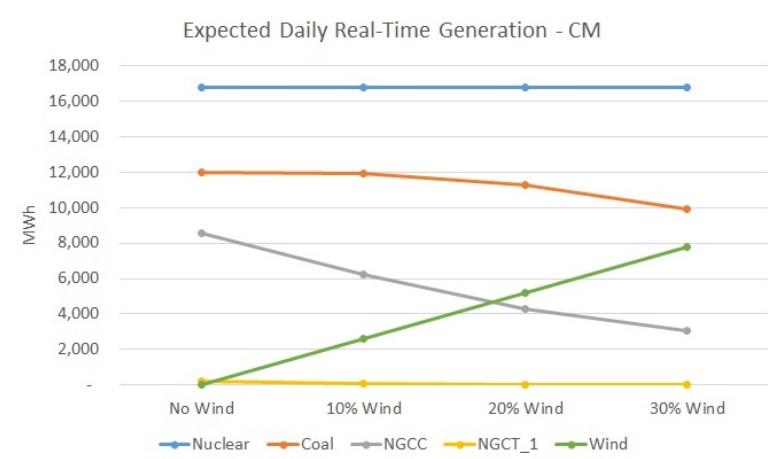

Figure 7. Expected Real-Time Generation - CM

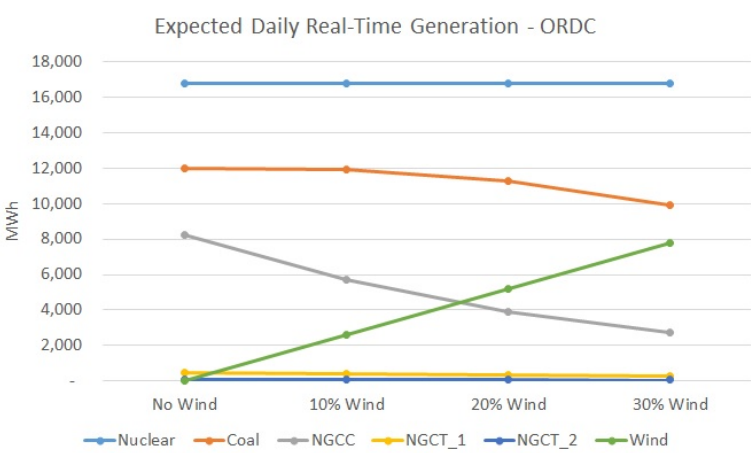

Figure 8. Expected Real-Time Generation - ORDC

To examine how resource sufficiency is affected as wind penetration increases, we compare annual carrying costs to total annual revenues (in $\$ / \mathrm{kW}-\mathrm{yr}$ ) in the capacity and energy markets. ${ }^{4}$ The annual carrying cost of a generator includes its annualized fixed costs, which are based on the capital costs of building the plant, and its annual operating costs, which depend on the total usage of the plant. The carrying cost represents the total annual cost a power plant would need to recover through the capacity, energy and reserve markets. Figures 9 - 13 compare the annual carrying costs to the annual revenues for each generator (except the coal plant, whose trends are similar to the nuclear plant) and each wind case. It should be noted that carrying costs may be at different levels, depending on plant usage under each wind case. For example, generation from the NGCC plant starts out high in the no wind case, but decreases as wind penetration increases. This causes the annual operating costs to decrease, which in turn leads to a decrease in the total annual carrying costs.

Revenues for the nuclear, coal, and NGCC plants drop significantly under both market structures, as wind

\footnotetext{
${ }^{4}$ Results presented here assume a moderate level of electricity demand. Thus, there is no reserve scarcity in any time period, i.e. reserve prices are always zero and plants do not receive reserve revenues.
} 
penetration increases in the system. The NGCT 1 plant shows only a small decrease in revenue in the capacity market structure, but revenue decrease is sharper in the energy-only market structure. Notably, for CT 1 revenues in the energy-only market are higher than in the capacity market model in the no wind and 10\% wind cases, but lower in the $20 \%$ and $30 \%$ cases. This can be explained by a quantity effect. In the no wind and $10 \%$ wind cases of the energy-only market model, NGCT 1 produces enough electricity to make revenues higher than the combined capacity and energy revenues in the capacity market design. In the 20\% and 30\% wind cases of the energy-only market model, production from NGCT 1 decreases and revenues drop below the capacity market revenues. The NGCT 2 plant doesn't produce in the capacity market model, therefore all revenues come from the capacity market. In contrast, this unit has non zero generation under the energy-only market structure, but does not produce enough to match revenues in the capacity market structure. Finally, the wind plant always produces at its forecast levels, but as wind penetration increases, its revenues decrease due to lower energy prices.

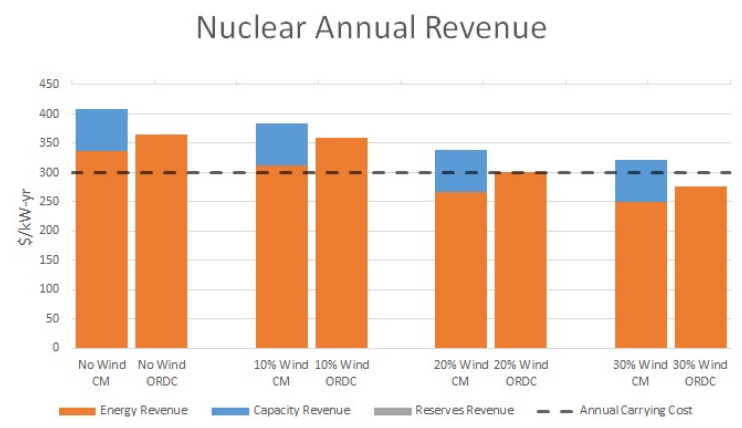

Figure 9. Annual Revenue and Carrying Cost Comparison - Nuclear

NGCC Annual Revenue

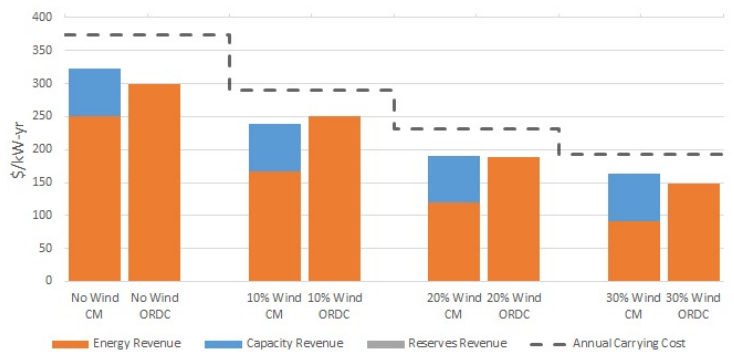

Figure 10. Annual Revenue and Carrying Cost Comparison - NGCC

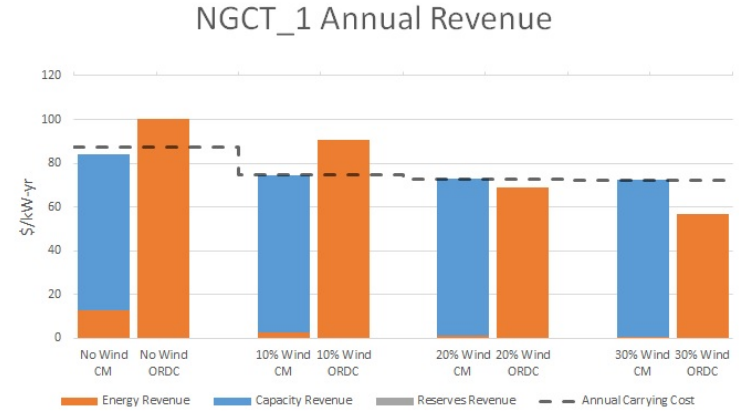

Figure 11. Annual Revenue and Carrying Cost Comparison - NGCT 1

\section{NGCT_2 Annual Revenue}

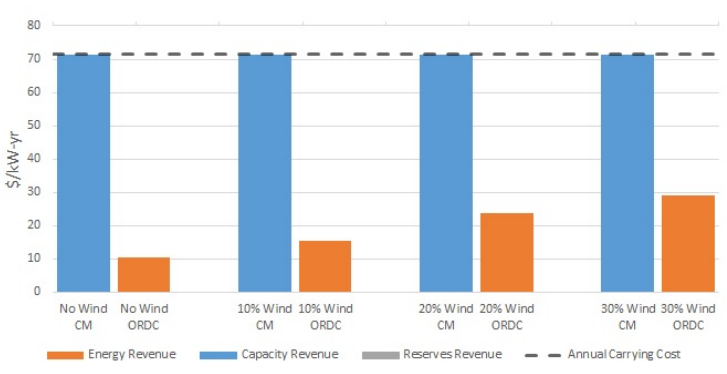

Figure 12. Annual Revenue and Carrying Cost Comparison - NGCT 2

Wind Annual Revenue

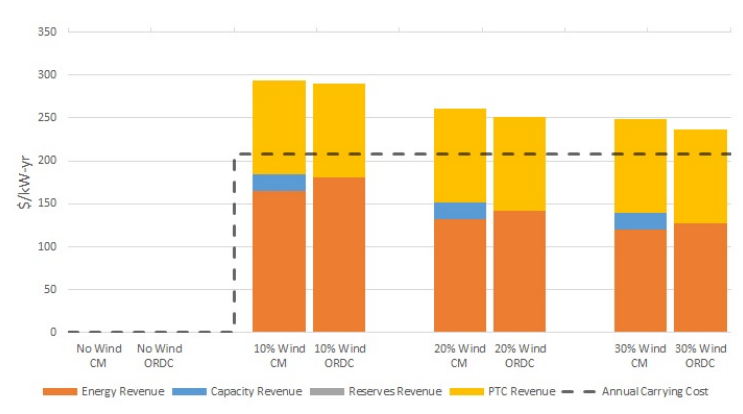

Figure 13. Annual Revenue and Carrying Cost Comparison - Wind

\section{Conclusions}

We analyze the performance of capacity markets and energy-only markets under increasing wind penetration, and uncertainty in wind generation and natural gas prices. First, we formulate a three-stage equilibrium model (for the capacity market design) and a nonlinear optimization model (for the energy-only market design with ORDC) that determine optimal capacity expansion decisions, and hourly commitment 
and dispatch decisions. Second, we present impacts of increasing wind penetration under uncertainty on capacity expansion and generator profitability in an ideal seven node network. Preliminary results suggest that: a) the least generation capacity is added in the ORDC framework; b) as wind penetration increases, generator revenues decrease more for baseload units (nuclear, coal, NGCC) than for peak units, and c) under no reserve scarcity, an energy-only market structure with a dynamic ORDC does not guarantee revenue sufficiency. Next steps include simulating scenarios that include reserve scarcity, reformulating the energy-only market design as an equilibrium problem, including additional metrics to assess resource adequacy, and adding exit decisions to the model formulation. We also plan on solving the models on a test system with more realistic topology (i.e., a modified version of the 36-bus electric network in [25]), and selecting representative days in a year to more accurately determine the probability of moving from the capacity to the day-ahead market stage. In the 36-bus test system, wind day-ahead forecasts and real-time output will be based on locational data from NREL.

\section{References}

[1] P. L. Joskow, "Capacity Payments in Imperfect Electricity Markets: Need and Design," Utilities Policy, vol. 16, pp. 159-170, 2008.

[2] D. Hach, C. Chyong, and S. Spinler, "Capacity Market Design Options: A Dynamic Capacity Investment Model and a GB Case Study," European Journal of Operations Research, vol. 249, no. 2, pp. 691-705, 2016.

[3] C. Vazquez, M. Rivier, and I. Perez-Arriaga, "A Market Approach to Long-term Security of Supply," IEEE Transactions on Power Systems, vol. 17, no. 2, pp. 349-357, 2002.

[4] S. Oren, "Generation Adequacy via Call Options Obligations: Safe Passage tot the Promised Land," The Electricity Journal, vol. 18, no. 9, pp. 28-42, 2005.

[5] C. Byers, T. Levin, and A. Botterud, "Capacity Market Design and Renewable Energy: Performance Incentives, Qualifying Capacity and Demand Curves," The Electricity Journal, vol. 31, pp. 65-74, 2018.

[6] W. W. Hogan, "Electricity Scarcity Pricing Through Operating Reserves," Economics of Energy $\mathcal{G}$ Environmental Policy, vol. 2, no. 2, pp. 65-86, 2013.

[7] Federal Energy Regulatory Commission, "Price Formation in Organized Wholesale Electricity Markets: Staff Analysis of Shortage Pricing in RTO and ISO Markets." https://www. ferc.gov/legal/staff-reports/2014/ AD14-14-pricing-rto-iso-markets .pdf, 2014.

[8] Entergy, "Market Challenges Facing Competitive Power Markets in the Northeast U.S.." http: //www.ncsl.org/Portals/1/Documents/energy/ Mohl-present.pdf, 2015.
[9] M. Cepeda and D. Finon, "Generation Capacity Adequacy in Interdependent Electricity Markets," Energy Policy, vol. 39, no. 6, pp. 3128-3143, 2011.

[10] D. F. M. Petitet and T. Janssen, "Capacity Adequacy in Power Markets Facing Energy Transition: a Comparison of Scarcity Pricing and Capacity Mechanism," Energy Policy, vol. 103, pp. 30-46, 2017.

[11] N. Dahlan and D. Kirschen, "Generation Investment Evaluation Model in Electricity Market with Capacity Mechanisms," International Review of Electrical Engineering, vol. 9, no. 4, pp. 844-853, 2014.

[12] T. Levin and A. Botterud, "Electricity Market Design for Generator Revenue Sufficiency with Increased Variable Generation," Energy Policy, vol. 87, pp. 392-406, 2015.

[13] P. Mastropietro, I. Herrero, P. Rodilla, and C. Batlle, "A Model-Based Analysis on the Impact of Explicit Penalty Schemes in Capacity Mechanisms," Applied Energy, vol. 168, pp. 38-47, 2016.

[14] D. Keles, A. Bublitz, F. Zimmermann, M. Genoese, and W. Fichtner, "Analysis of Design Options for the Electricity Market: The German Case," Applied Energy, vol. 183, pp. 884-901, 2016.

[15] P. Bhagwat, K. Iychettira, J. Richstein, E. Chapping, and L. D. Vries, "The Effectiveness of Capacity Markets in the Presence of a High Portfolio Share of Renewable Energy Sources," Utilities Policy, vol. 48, pp. 76-91, 2017.

[16] A. Haurie, G. Zaccour, and Y. Smeers, "Stochastic Equilibrium Programming for Dynamic Oligopolistic Markets," Journal of Optimization Theory and Applications, vol. 66, no. 2, pp. 243-253, 1990.

[17] A. Papavasiliou and Y. Smeers, "Remuneration of Flexibility using Operating Reserve Demand Curves: A Case Study of Belgium," The Energy Journal, vol. 38, no. 6, pp. 105-135, 2017.

[18] PJM Interconnection, "Wind Generation Operating Reserves." https://pjm.com/Globals/Training/ Courses/ol-wind. aspx, 2019.

[19] Electricity Reliability Council of Texas, "Methodology for Implementing Operating Reserve Demand Curve (ORDC) to Calculate Real-Time Reserve Price Adder." http://www. ercot. com/mktinfo/rtm/ kd/Methodology_for_Implementing_Operating_ Reserve_Demand_Curve_.doc, 2013.

[20] PLEXOS, "Optimal Power Flow," tech. rep., PLEXOS, 2009.

[21] SNL Energy, "S \& P Global Market Intelligence,” 2018.

[22] EIA, "An Analysis of Nuclear Power Plant Construction Costs," tech. rep., Energy Information Administration, 1986.

[23] J. McNerney, J. Farmer, and J. Trancik, "Historical costs of coal-fired electricity and implications for the future," Energy Policy, vol. 39, pp. 3042-3054, 2011.

[24] R. Tidball, J. Bluestein, N. Rodriguez, and S. Knoke, "Cost and Performance Assumptions for Modeling Electricity Generation Technologies," tech. rep., NREL, 2010.

[25] E. Allen, J. Lang, and M. Ilic̀, "A Combined Equivalenced-Electric, Economic and Market Representation of the Northeastern Power Coordinating Council U.S. Electric Power System," IEEE Transactions on Power Systems, vol. 23, no. 3, pp. 896-907, 2008. 